\title{
NANOELECTROMECHANICAL SYSTEMS: POTENTIAL, PROGRESS, \& PROJECTIONS
}

\author{
M. L. Roukes \\ Kavli Nanoscience Institute and Departments of Physics, Applied Physics, and Bioengineering \\ California Institute of Technology, Pasadena, CA 91125 USA \\ Telephone: +1-626-395-2916 Fax: +1-626-683-9060 E-mail: roukes@ caltech.edu
}

\begin{abstract}
Nanoelectromechanical systems (NEMS) represent the next regime of size reduction beyond the microscale for mechanical devices. In their tiniest, ultimate realization, NEMS will be formed with sub-nanometer scale precision from atomic- and molecular-scale mechanical elements as first envisaged by Feynman (1). Although nanowire and nanotube based NEMS today verge on this domain, their assembly into functional devices remains more of an art than a science, as they are typically fabricated one-by-one by complicated means with low yield. By contrast, the most robust forms of NEMS are currently patterned by top-down methods; in fact their production is now being scaled to enable large-scale integration over $200 \mathrm{~mm}$ wafers with minimum feature sizes that are below $50 \mathrm{~nm}$.
\end{abstract}

In this paper I will describe how nanoscale mechanical elements provide benefits beyond the obvious, that is, benefits in addition to the possibility of increased device density. The reduced size of NEMS enables mechanical functionality that completely transcends what is possible at the microscale with MEMS (2). However, size reduction to the nanoscale may not be a panacea for all applications - for some applications larger may still be better!

\section{INTRODUCTION}

Many of the most compelling applications of NEMS involve resonant devices. These can be realized as two distinct classes of structures: as beam-like devices with large lengthto-cross-section ratios, or as devices with more compact boxlike or thick-plate geometries. For devices of both classes, as their size is scaled downward their resonant frequencies move upward. For the smallest devices, these move into the low (Lto C-band) microwave regime. This opens exciting new frontiers for sensing (3) and signal processing (4).

The first class, NEMS with beam geometries, especially excel at sensing. With beam-like devices high frequencies can be attained while "responsivities" are also simultaneously scaled upward; this is described in the next section. A large responsivity, in turn, immensely simplifies the otherwise difficult task of obtaining optimal read-out of the mechanical response to applied input signals. Ideally, a mechanical system's noise floor - that is, its minimum resolvable signal is set by mechanical-domain noise induced by thermal (3) or, at the ultimate limit, quantum fluctuations (5). Conventionally-applied methodologies from MEMS are generally incapable of providing such performance - i.e. of sensitivity limited solely by fundamental noise processes at high frequencies. Recently-developed NEMS transduction techniques make this possible (6).

The second class of devices, having more compact geometries (such as FBARs, wine-glass resonators, etc.), operate at high frequency with significantly "stiffer" modes. For these devices, UHF and microwave frequencies are attainable even at the low micron dimensions, although nanoscale features for transducers often become imperative. Their higher mode stiffness has the benefit of enabling higher power handling than is possible with beam-like NEMS, and this can make them more compatible with the signal levels employed in conventional signal processing electronics. The downside, however, is that they cannot provide the immense responsivities attainable with beam-like NEMS and, thus, prove inferior for many sensing applications.

Here we'll focus on the former - highlighting some of the exciting advances that are now being made with beam-like NEMS sensors.

\section{MECHANICAL PERFORMANCE AND SCALING}

If all the dimensions of a beam-like NEMS resonator are uniformly scaled downward, its frequency will scale upward with its inverse linear dimension, $1 / \ell$. Simultaneously its mechanical responsivity - that is its compliance to externallyapplied forces, $1 / K_{e f f}^{(\mathrm{n})}$ (here $K_{\text {eff }}^{(\mathrm{n})}$ is the modal force constant) - will also scale upward with $1 / \ell$. Meanwhile, ideally, the spectral density of the resonator's thermodynamic force fluctuations in the mechanical domain for the $\mathrm{n}^{\text {th }}$ mode, $S_{F}^{(\mathrm{n})}(\omega)=4 k_{B} T \gamma_{(\mathrm{n})}$ establish the attainable force resolution for sensing with that mode. This is directly proportional to the mechanical damping, which, in vacuum, can be expressed as $\gamma_{(\mathrm{n})}=K_{e f f}^{(\mathrm{n})} /\left(\omega_{(\mathrm{n})} Q_{(\mathrm{n})}\right)$. Hence the force noise floor scales downward with device size as $\ell^{2}$, if $Q$ is maintained. It is the simultaneous lowering of the device compliance with increased frequency makes the beam-like NEMS structures 
unique for sensing.

For applications beyond force sensing, other responsivities become important. An especially compelling attribute of NEMS resonators is their unprecedented mass responsivity, which, for the fundamental mode is $\Re=\partial \omega_{0} / \partial M_{\text {eff }}=-\omega_{0} /\left(2 M_{\text {eff }}\right)$. Here $\omega_{0}$ and $M_{\text {eff }}$ are the resonant frequency and effective mass of the fundamental mechanical mode. For uniform reduction a simple cantilever with linear dimension $\ell$, one finds that $\mathfrak{R} \propto 1 / \ell^{4}$. Hence, even making what might at first seem only a minor transition from the micro- to nanoscale, a truly profound sensitivity improvement is obtained (6). For devices with sizes comparable to the mean free path in air, the added benefit of preservation of a high quality factor in ambient enables unprecedented, sub-attogramscale mass resolution at room temperature and atmospheric pressure, as recently demonstrated (6). These attributes make self-sensing nanometer-scale resonators a surprisingly versatile and promising platform for chemical, biological, and scanned-probe sensing.

\section{ATTAINING OPTIMAL TRANSDUCTION}

For NEMS and MEMS devices, their thermodynamic displacement fluctuations on resonance, $S_{x}^{1 / 2}\left(\omega_{(\mathrm{n})}\right)=S_{x}^{1 / 2}\left(\omega_{(\mathrm{n})}\right)\left[Q / K_{\text {eff }}\right]$, are usually quite small. The stiffer the device, the smaller will be the transduced noise floor in the displacement domain. High frequency NEMS can require extreme sub-nanometer scale resolution with large bandwidth to attain fundamental limits of performance. This is a second reason why beam-like NEMS excel for sensing applications, given their higher mechanical compliance, over the more compact geometries.

\section{NONLINEAR NEMS}

The onset of nonlinearity - crucial for many classes of switching applications and for parametric processes - occurs for smaller applied force (hence lower input power) in beamlike NEMS. In fact, for very large aspect ratio doublyclamped structures we have recently shown that the linear dynamic range, bounded by the thermomechanical noise floor and the onset of nonlinearity, can vanish - meaning that nanotube resonators are intrinsically nonlinear elements (7).

We have recently employed the easily-attainable nonlinear response of beam-like NEMS to realize efficient tuning of the frequency and nonlinearity of NEMS resonators. The Euler instability is another mechanical nonlinearity that provides opportunity for all-mechanical parametric gain (8).

\section{COLLECTIVE-MODE NEMS}

Compelling applications are certain to come from "ganging" the response of individual NEMS elements to realize complex functionality. Beyond the obvious possibilities of engineering arbitrary filter response at microwave frequencies in a compact NEMS format, opportunities are emerging with nonlinearly coupled NEMS arrays for applications that include enhanced noise averaging, acoustic logic, and bifurcation amplification (8).

\section{PROJECTIONS}

The ultimate limits of NEMS are at the molecular or atomic scale - and there the frequencies and time scales are set by the vibrational properties of molecules. First precursors of the future era of molecular mechanical systems are the buckyball, nanotube-, and nanowire-based resonators that are currently being explored in various laboratories - albeit at the level of "heroic" individual devices.

Ultimately it seems that efforts in molecular electronics and NEMS will meld in pursuit of future electronic paradigms, and for sensing and information processing systems. Both approaches currently rely, explicitly, upon mechanical degrees-of-freedom (be they vibrations or conformations) to derive their functionality, i.e. to achieve controllable device "states" upon which specific functions can be based. This rich field is certainly in its infancy.

\section{REFERENCES}

(1) R.P. Feynman, "Plenty of Room at the Bottom", Presentation to American Physical Society, Dec. 29, 1959 California Institute of Technology, Pasadena, CA; available online at: http: //www.its.caltech.edu/ feynman/

(2) M.L. Roukes, "Plenty of Room, Indeed", Scientific American, Special Edition: "The Edge of Physics" (Jan. 2003); ibid. 285, 48-57 (Sept. 2001).

(3) M.L. Roukes, "Nanoelectromechanical Systems," Technical Digest of the 2000 Solid-State Sensor and Actuator Workshop, Cleveland: Transducer Research Foundation, 2000; available online at http://arxiv.org/pdf/cond-mat/0008187

(4) C.T.-C. Nguyen, "Frequency-selective MEMS for miniaturized low-power communication devices", IEEE Trans MTT 47, 1486-1503 (1999).

(5) K.C. Schwab and M.L. Roukes, "Putting mechanics into quantum mechanics”, Physics Today 58,: 36-42 (July 2005).

(6) M. Li, H.X. Tang, and M.L. Roukes, "Ultra-sensitive NEMS-based cantilevers for sensing, scanned probe and VHF applications", Nature Nanotechnology, in press (February 2007).

(7) H.W.C. Postma, I. Kozinsky, A. Husain, M.L. Roukes, "Dynamic range of nanotube- and nanowire-based electromechanical systems", Appl. Physics Lett. 86, Art. No. 223105 (2005).

(8) R. Karabalin, S. Masmanidis, M.L. Roukes, to be published (2007). 